\title{
Multiple ionization of argon via multi-XUV-photon absorption induced by 20-GW high-order harmonic laser pulses
}

\author{
A. Nayak, ${ }^{1}$ I. Orfanos, ${ }^{1,2}$ I. Makos, ${ }^{1,2}$ M. Dumergue, ${ }^{3}$ S. Kühn, ${ }^{3}$ E. Skantzakis, ${ }^{1}$ B. Bodi, ${ }^{4}$ K. Varju,${ }^{3,5}$ C. Kalpouzos, ${ }^{1}$ \\ H. I. B. Banks, ${ }^{6}$ A. Emmanouilidou, ${ }^{6}$ D. Charalambidis,,${ }^{1,2,3, *}$ and P. Tzallas ${ }^{1, *}$ \\ ${ }^{1}$ Foundation for Research and Technology-Hellas, Institute of Electronic Structure and Laser, P.O. Box 1527, GR71110 Crete, Greece \\ ${ }^{2}$ Department of Physics, University of Crete, P.O. Box 2208, GR71003 Crete, Greece \\ ${ }^{3}$ ELI-ALPS, ELI-Hu Kft., Dugonics ter 13, H-6720 Szeged, Hungary \\ ${ }^{4}$ MTA “Lendület” Ultrafast Nanooptics Group, Wigner Research Center for Physics, 1121 Budapest, Hungary \\ ${ }^{5}$ Department of Optics and Quantum Electronics, University of Szeged, Szeged, Hungary \\ ${ }^{6}$ Department of Physics and Astronomy, University College London, Gower Street, London WC1E 6BT, England, United Kingdom
}

(Received 4 May 2018; published 27 August 2018)

\begin{abstract}
We report the observation of multiple ionization of argon through multi-XUV-photon absorption induced by an unprecedentedly powerful laser driven high-order harmonic generation source. Comparing the measured intensity dependence of the yield of the different argon charge states with numerical calculations we can infer the different channels-direct and sequential—underlying the interaction. While such studies were feasible so far only with free electron laser (FEL) sources, this paper connects highly nonlinear XUV processes with the ultrashort time scales inherent to the harmonic pulses and highlights the advanced perspectives of emerging large scale laser research infrastructures.
\end{abstract}

DOI: 10.1103/PhysRevA.98.023426

\section{INTRODUCTION}

Multiphoton processes trace back to 1931 [1]. One third of a century later, the invention of lasers has allowed their observation [2], followed by several decades of flourishing multiphoton and strong-field science. Another third of a century later two-photon processes made their debut in the XUV spectral region [3], paving the way to XUV-pump and XUV-probe studies in the 1-fs scale [4] and below. Instrumental to these studies is high-order harmonic generation (HHG), which apart from its importance in understanding strongfield laser-atom interactions [5-7] led to the development of coherent XUV sources with pulse durations in the attosecond regime [8-14]. While HHG and attosecond sources induced so far few-photon (mainly two-photon) processes, FEL sources have achieved the production of high charge states through absorption of many XUV [15] or X-ray [16-18] photons. Attaining insight into such processes is considered of central importance [15-18], as they govern a wide-ranging spectrum of applications of energetic XUV and X-ray sources. Multiple multi-XUV-photon ionization by harmonic and attosecond sources provides an advanced tool for the study of ultrafast dynamics in correlated systems and/or of coupled motions due to the unprecedentedly short duration of their pulses. Towards this goal a substantial increase of their pulse energy is required. Despite FELs' markedly higher pulse energy (hundreds of $\mu \mathrm{J}$ at $\sim 60 \mathrm{~nm}$ ) [19], their capacity in ultrafast XUV science is determined by their so far longer pulse durations [20] and shot-to-shot instabilities. Consequently, the enhancement of the HHG pulse energy in parallel with the reduction of the

*Corresponding authors: chara@iesl.forth.gr; ptzallas@iesl.forth.gr
FEL pulse duration remain challenging tasks serving the same goal.

Here, using the high-order harmonics generated by the interaction of high-power IR femtosecond laser pulses with xenon (Xe) and argon (Ar), we demonstrate a 20-GW XUV source which delivers pulses with carrier wavelength $\lambda_{\mathrm{XUV}} \approx 50 \mathrm{~nm}$ and pulse energy $E_{\mathrm{XUV}} \approx 230$ and $130 \mu \mathrm{J}$, respectively. Using this source, highly charged $\mathrm{Ar}$ ions (up to $\mathrm{Ar}^{+4}$ ) have been observed (an accomplishment that was up to now feasible only with FEL sources [21]) and the process of multiple ionization of Ar has been investigated by measuring the dependence of the multiply charged ions on the intensity of the XUV pulses.

\section{EXPERIMENTAL PROCEDURE}

The 20-GW XUV source is based on the increase in the number of the XUV emitters using loose IR focusing geometry and the precise control of phase-matching conditions achieved by means of thin single-gas targets in a dual-jet configuration with controllable distance between the jets. The $\approx 18$-m-long beam line [Fig. 1(a)] was recently developed in the Attosecond Science and Technology laboratory of the Foundation of Research and Technology (FORTH). It is driven by a $10-\mathrm{Hz}$ repetition rate Ti:sapphire laser system, which delivers $\tau_{L} \approx$ 20 -fs pulses at 800 -nm carrier wavelength (IR) and energy up to $\approx 400 \mathrm{~mJ} /$ pulse. A $p$-polarized IR pulse of $25-45-\mathrm{mJ}$ energy is focused by a spherical mirror of 9-m-long focal length into the HHG area which hosts a dual-pulsed-jet (GJ1,2) configuration operated with the same noble gas (either Ar or $\mathrm{Xe}$ ) as nonlinear medium. In the dual gas-jet configuration, the gas pressure and the medium length were the same for both gas jets. This was confirmed by measuring the same harmonic yield generated by the individual jets when they were 


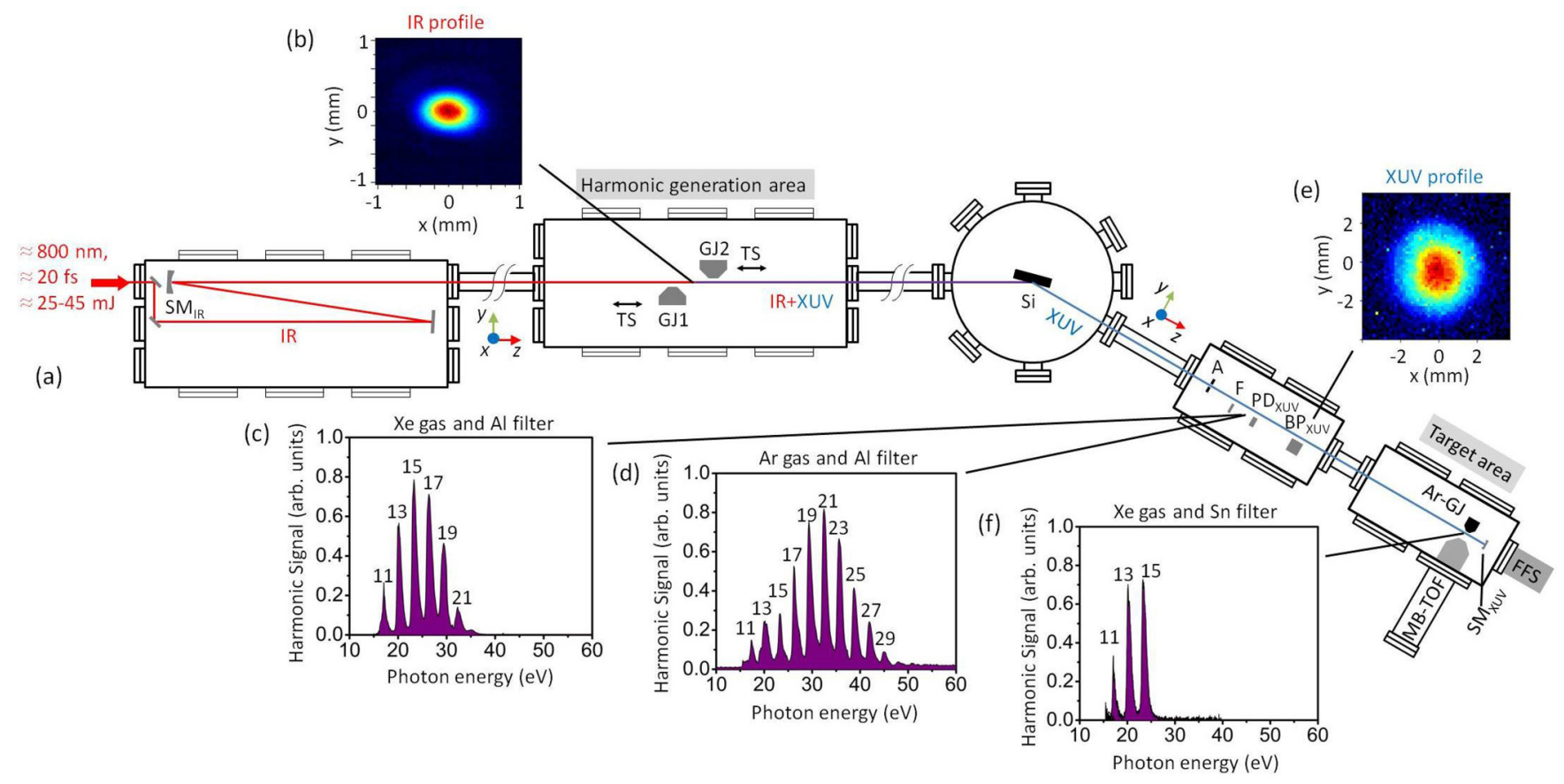

FIG. 1. (a) A drawing of the 20-GW XUV beam line. $\mathrm{SM}_{\mathrm{IR}}$ : Spherical mirror of 9-m focal length. GJ1,2: Dual-pulsed-jet configuration placed on translation stages (TS). Si: Silicon plate. F: Al or Sn filter. $\mathrm{BP}_{\mathrm{XUV}}$ : XUV beam profiler. $\mathrm{SM}_{\mathrm{XUV}}$ : Gold coated spherical mirror of 5-cm focal length. Ar-GJ: Ar gas jet. MB-TOF: Magnetic bottle time-of-flight spectrometer. PD $_{\mathrm{XUV}}$ : Calibrated XUV photodiode. FFS: Flat-field spectrometer. (b) IR beam profile at the focus. (c), (d) Harmonic spectra generated in Xe and Ar gases and transmitted by the Al filter. (e) XUV beam profile. (f) Harmonic spectrum generated in Xe gas transmitted by the Sn filter.

placed at the same position relative to focus of the IR beam. The slit shaped orifice of the piezo-based pulse nozzle has dimensions $0.3 \times 2 \mathrm{~mm}$. The pressure and the medium length were estimated taking into account the backing pressure of the nozzle, the conductance of the orifice, and the distance between the orifice and the laser focus, which was $\sim 1 \mathrm{~mm}$. The values are in agreement with those reported in Ref. [22] where piezo-based pulsed nozzles have been used. The gas jets were placed on $x, y$, and $z$ translation stages with the movement on the $x$ and $y$ axes being manually controlled, while the displacement along the propagation $z$ axis was done by a motorized translation stage using $\approx 0.75-\mathrm{cm}$ steps (the minimum step of the stage was $5 \mu \mathrm{m}$ ). The IR beam profile in the HHG area is shown in Fig. 1(b). A silicon ( $\mathrm{Si}$ ) plate, placed after the harmonic generation at the Brewster angle for the fundamental (i.e., $75 \mathrm{deg}$ ), reflects the harmonics towards the detection area, while substantially attenuating the IR field. The reflectivity of the Si plate is $\approx 60 \%$ in the spectral range of 15 to $45 \mathrm{eV}$ [23]. After reflection from the Si plate, the XUV radiation passes through a 5-mm-diameter aperture (A) which blocks the residual outer part of the IR beam. The harmonics used in the experiments were selected by means of thin metal filters. A tin (Sn) filter with $\approx 20 \%$ transmission in the spectral range $17-23 \mathrm{eV}$ was used for studying the multiple ionization of Ar, while for the measurement of the XUV energy and XUV beam profile a low transmission $(\approx 5 \%$ in the spectral range $17-60 \mathrm{eV}$ ) aluminum (Al) filter was used in order to avoid damaging and/or saturating the detectors. The transmission of the filters was measured by recording the harmonic spectra with and without the filters. Also, the transmission of the IR beam after the metal filters was negligible. This was confirmed by the zero response of the $\mathrm{BP}_{\mathrm{XUV}}$ (consisting of a pair of multichannel plates (MCPs) and a phosphor screen) and PD $_{\text {XUV }}$ (XUV calibrated photodiode) detectors (both sensitive to the IR radiation) after blocking the XUV beam by a 2-mmthick BK7 window. The harmonic spectra were measured by a flat-field spectrometer (FFS) attached to the back side of the target area chamber. The profile of the XUV beam was recorded by the $\mathrm{BP}_{\mathrm{XUV}}$ placed after the $\mathrm{Al}$ filter. The harmonic spectra generated in Xe and Ar gases and the XUV beam profile after the Al filter are shown in Figs. 1(c)-1(e), respectively. For the studies of the multiple ionization of Ar, the beam transmitted through the Sn filter was then focused by a gold coated spherical mirror $\left(\mathrm{SM}_{\mathrm{XUV}}\right.$ positioned at $\sim 2^{\circ}$ angle of incidence) of 5-cm focal length into an Ar gas jet (Ar-GJ) placed in the target area. The harmonic spectrum at the position of the Ar-GJ is shown in Fig. 1(f). The ionization products were measured by a magnetic bottle time-of-flight (MB-TOF) spectrometer that can be set to record either the photoelectron energy (PE) distribution or the ion-mass spectrum. In some cases the harmonics passing through the filters have been, for convenience, also measured by recording the single-photon $\mathrm{Ar}$ ionization PE spectra produced by the incoming (unfocused) harmonic beam. The intensity of the XUV radiation is changed by changing the atomic density of the harmonic generation target through variation of the delay between the laser and the gas nozzle trigger pulse. The reflectivity of the gold mirror $(\approx 12 \%)$ is constant in the spectral region of $17-23 \mathrm{eV}$ [24]. We note that in the spectral range from 15 to $30 \mathrm{eV}$, the measured photoelectron distribution does not differ significantly from 
the spectrum measured by the FFS as in this photon energy range the single-photon-ionization cross section of argon is almost constant at $\approx 30 \mathrm{Mb}$ [25]. The deviation (compared to the spectra recorded by the flat-field spectrometer) appearing at photon energies $>30 \mathrm{eV}$ is attributed to the reduction of the single-photon ionization cross section. The energy of the XUV radiation in the generation region and the target area was determined by means of $\mathrm{PD}_{\mathrm{XUV}}$, taking into account the transmission of the filters and the reflectivity of the XUV optics.

\section{PULSE ENERGY AND CONVERSION EFFICIENCY OF THE XUV SOURCE}

In gas-phase harmonics, the amount of the XUV energy exiting the gas medium is an interplay between the microscopic (single atom) and macroscopic (atomic ensemble) response of the medium. On the microscopic level, for a specific driving laser field wavelength $\left(\lambda_{\mathrm{L}}\right)$, the probability of the emission of a single XUV photon depends nonlinearly on the driving laser field intensity $\left(I_{\mathrm{L}}\right)$ and the atomic properties. Considering that the probability of the single-XUV-photon emission is maximized for a fixed $I_{\mathrm{L}}$ lying just below the ionization saturation threshold of the atom (which for xenon and argon atoms is $I_{\mathrm{L}}<3 \times 10^{14} \mathrm{~W} \mathrm{~cm}^{-2}$ ), it is evident that for the enhancement of the energy of the XUV radiation one has to increase the number of the atomic XUV emitters and consider the macroscopic response of the medium. While keeping $I_{\mathrm{L}}$, at the level of saturating single atom ionization the number of the emitters can be increased by increasing either the interaction volume (by increasing focal length together with laser pulse energy) or the atomic density of the medium. Incorporating the macroscopic response taking into account the propagation effects in the gas medium, it has been shown (see Refs. [26-28] and references therein) that for $L_{\mathrm{coh}} \gg L_{\text {abs }}$ and $L_{\text {coh }} \gg L_{\text {med }}$ the XUV yield is proportional to $\propto\left(\rho L_{\text {med }}\right)^{2}$. In the former expressions $L_{\mathrm{coh}}=\pi / \Delta k, L_{\mathrm{abs}}=1 / \rho \sigma^{(1)}$, and $L_{\text {med }}$ are the coherence length, the absorption length of the XUV radiation, and the gas medium length, respectively, with $\Delta k=k_{\mathrm{L}}-q k_{\mathrm{L}}$, $q$ the harmonic order, $k_{\mathrm{L}}$ the wave number of the driving field, $\rho$ the atomic density of the medium, and $\sigma^{(1)}$ the single-XUVphoton ionization cross section of the atoms in the medium. This product constitutes the main scaling factor towards the enhancement of the produced energy. Using one gas jet, for fixed $I_{\mathrm{L}}$ the dependence of the harmonic yield on gas pressure $(P)$ and $L_{\text {med }}$ is shown in the contour plot of Fig. 2. The red color area corresponds to the area of maximum XUV production and the black-circled area depicts the values of $P$ and $L_{\text {med }}$ used in the present paper. The $\sim 50 \%$ reduction of the XUV emission for "large"-length and "high"-pressure media (green color area in Fig. 2) is associated with the XUV absorption effects and IR-XUV phase mismatch induced by the neutral atoms and plasma generation in the medium which confines the coherent harmonic buildup to a short propagation length. This limitation can be overcome applying quasi-phase-matching conditions discussed below.

The optimization of the generated energy in the dual-jet configuration was performed after maximizing the harmonic yield of the single-gas jet (GJ1). This was achieved by measuring the XUV energy as a function of the driving IR field

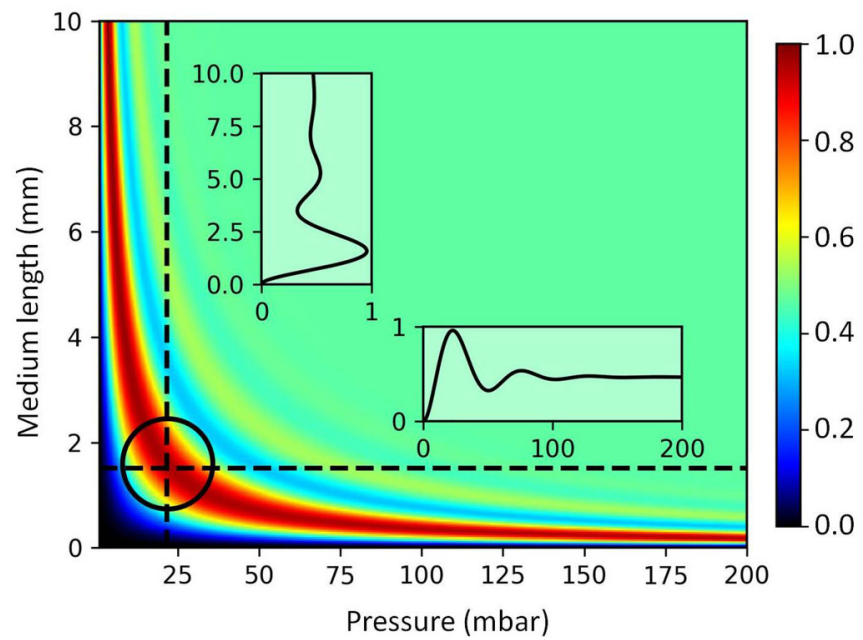

FIG. 2. Calculated harmonic yield generated in $\mathrm{Ar}$ gas as a function of the gas pressure $(P)$ and medium length $(L)$ for $I_{\mathrm{L}} \approx$ $1.5 \times 10^{14} \mathrm{~W} \mathrm{~cm}^{-2}$. The values of $P$ and $L$ used in the experiment fall within the area defined by the black circle. The insets show a line out of the harmonic yield along the dashed lines at $L \approx 1.5 \mathrm{~mm}$, $P \approx 25$ mbars.

intensity $\left(I_{\mathrm{L}}\right)$, the medium length $(L)$, the gas pressure $(P)$, and the position of the GJ1 relative to the focus position of the IR beam. The optimum conditions were found when GJ1 was set to be at the focus of the IR beam $(z=0)$ where the IR was just below the ionization saturation intensity of $\mathrm{Xe}$ and Ar atoms, for $P \sim 25$ mbars and $L \sim 1.5 \mathrm{~mm}$ [Figs. 3(a) and $3(\mathrm{~b})]$. These optimal conditions are in fair agreement with the results obtained by calculations [26-28] (Fig. 2). Further enhancement of the harmonic yield was achieved by applying quasi-phase-matching conditions [26,29] using two gas jets. GJ1 is positioned at fixed $z \approx 0$ and GJ2 at variable positions ( $L$ and $P$ are the same in both jets). The dependence of the XUV energy generated by $\mathrm{Xe}$ and $\mathrm{Ar}$ gas on the distance between GJ2 and GJ1 is shown in Figs. 4(a) and 4(b), respectively. In both cases, the energy increases by a factor of $\approx 1.7$ when GJ 2 is placed at $z \approx \pm 5 \mathrm{~cm}$, verified by calculations taking into account the propagation effects in the dual-gas medium [26] [Fig. 4(c)]. At this position, the generated XUV energy for Xe and $\mathrm{Ar}$ gas was $\approx 230$ and $130 \mu \mathrm{J}$ per pulse, respectively. The reduction of the energy around $z \approx 0$ is attributed to phase-mismatch effects induced due to the increase of the medium pressure and/or medium length, while the oscillations observed at $z>+5 \mathrm{~cm}$ and $z<-5 \mathrm{~cm}$ are due to the Gouy phase shift of the focused IR beam [30].

In order to estimate the conversion efficiency $\left(C_{(q)}=\right.$ $\left.E_{\mathrm{XUV}}^{(q)} / E_{\mathrm{IR}}\right)$ of the harmonic generation process the energy per harmonic $(q)$ per pulse $\left(E_{\mathrm{XUV}}^{(q)}\right)$ was calculated taking into account the transmission of the filter, the reflectivity of the $\mathrm{Si}$ plate, and the quantum efficiency of $\mathrm{PD}_{\mathrm{XUV}}$. For the single-jet configuration, and for the optimum generating conditions, it has been found that the maximum generated XUV energy (integrated over the spectrum) was $\approx 135$ and $75 \mu \mathrm{J}$ per pulse for Xe and Ar, respectively. This corresponds to $E_{\mathrm{XUV}}^{(q)}(q=11$, $13,15) \approx 30$ and $10 \mu \mathrm{J}$ for $\mathrm{Xe}$ and $\mathrm{Ar}$ gas, respectively, considering that the total energy is shared equally between 
(a)

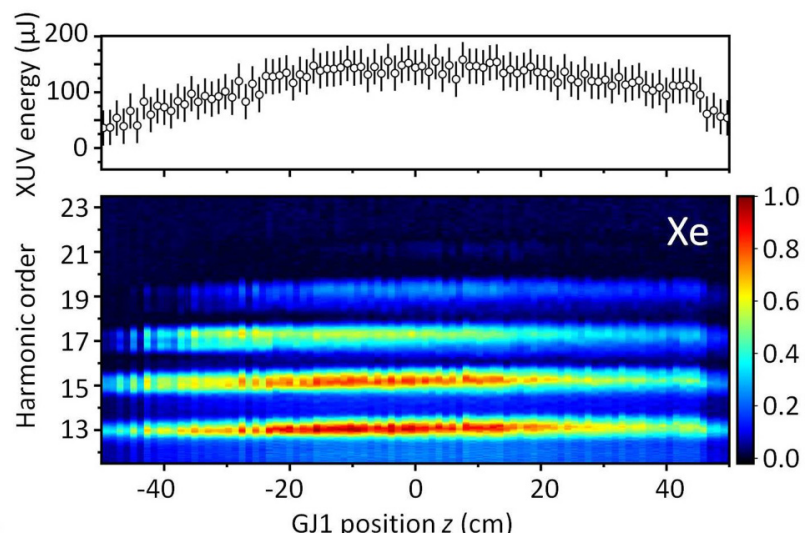

(b)

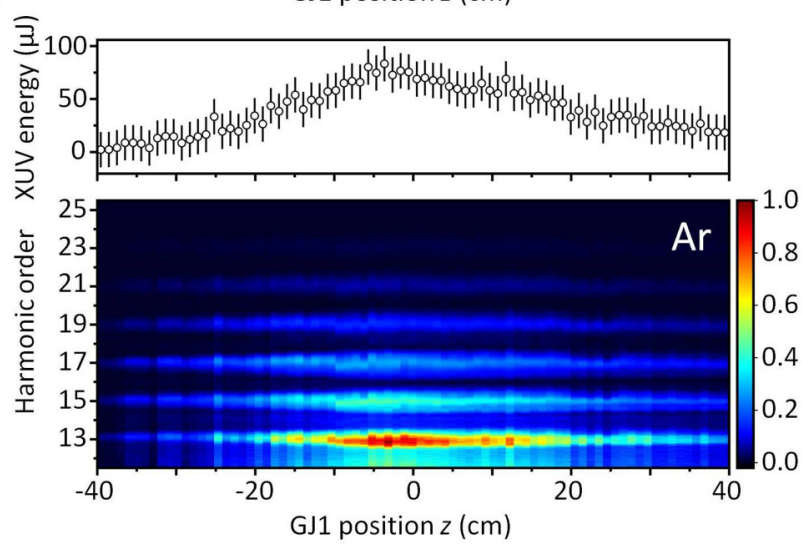

FIG. 3. (a), (b) The upper panels show the dependence of the XUV energy (integrated over the spectrum passing through the $\mathrm{Al}$ filter) generated in Xe and Ar gas on the position of the GJ1 relative to the laser focus, respectively. For obtaining the energy values just after the harmonic generation area, the measured by the $\mathrm{PD}_{\mathrm{xuv}}$ energy values were divided by the reflectivity of the Si plate and the transmission of the $\mathrm{Al}$ filter. The error bars represent one standard deviation from the mean. The lower panels show the corresponding harmonic spectrum measured in the target area by recording the single-photon PE spectra produced by the interaction of Ar gas with the incoming XUV beam.

the plateau harmonics. This results in $C_{(11)} \approx C_{(13)} \approx C_{(15)} \approx$ $1 \times 10^{-3}$ and $\approx 2 \times 10^{-4}$, respectively. The enhancement by a factor of $>2$ as compared to previously reported values [31] (where $C_{(11)} \approx 5 \times 10^{-4}$ ) is associated with the increased interaction volume and is in agreement with the energy scaling law reported in Refs. [28,32]. The dual-jet configuration provides a factor of $\approx 1.7$ further enhancement, resulting in a conversion efficiency $\approx 2 \times 10^{-3}$ and $\approx 3 \times 10^{-4}$, for Xe and $\mathrm{Ar}$, respectively.

\section{MUTIPLE IONIZATION OF ARGON}

Taking into account the measured XUV pulse energy and a focal spot size of $\approx 2 \mu \mathrm{m}[33]$, when low $(\approx 90 \%)$ energy loss XUV optics are used, this radiation can support trains of attosecond pulses with overall duration $\tau_{\mathrm{XUV}}=\tau_{L} / \sqrt{n} \approx 10 \mathrm{fs}$ (where $n=3-5$ is the order of nonlinearity of the generation of plateau harmonics [34]) and $I_{\mathrm{XUV}}$ up to $\sim 10^{17} \mathrm{~W} \mathrm{~cm}^{-2}$. However, in the present paper, for the investigation of multiple ionization process of Ar atoms the spectral region from 17.05 to $23.25 \mathrm{eV}$ was selected [Fig. 1(f)] using XUV optical elements (a)

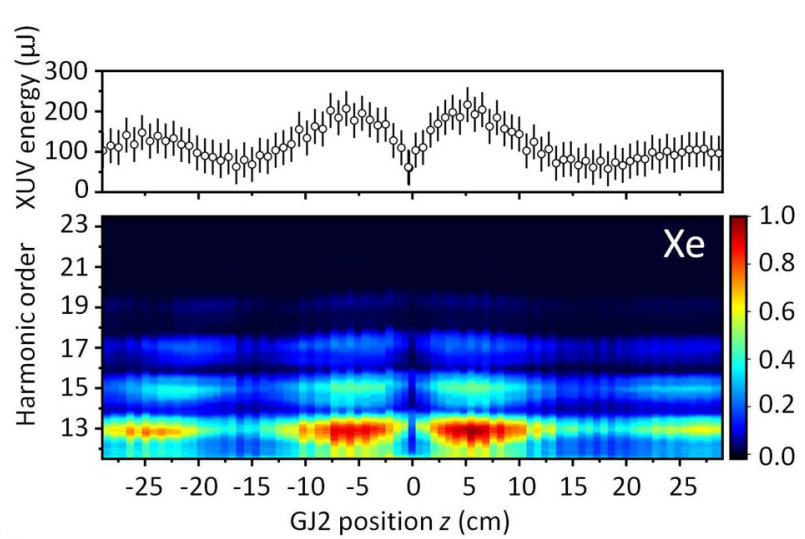

(b)

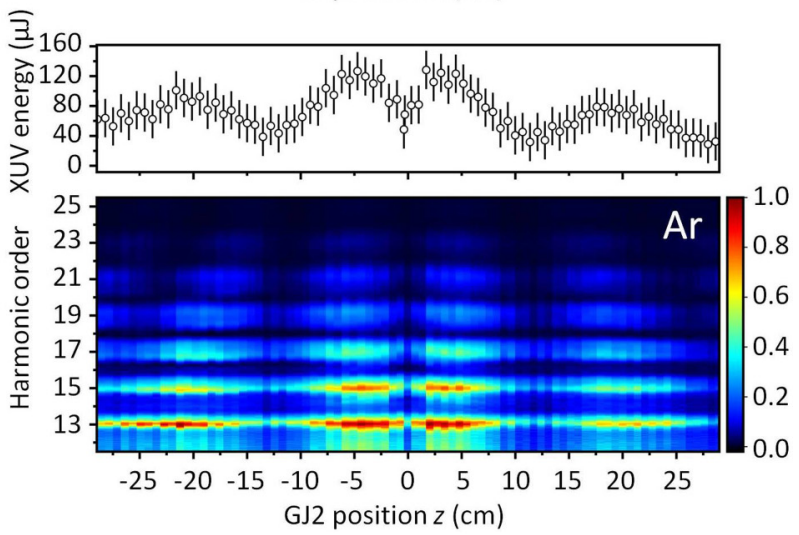

(c)

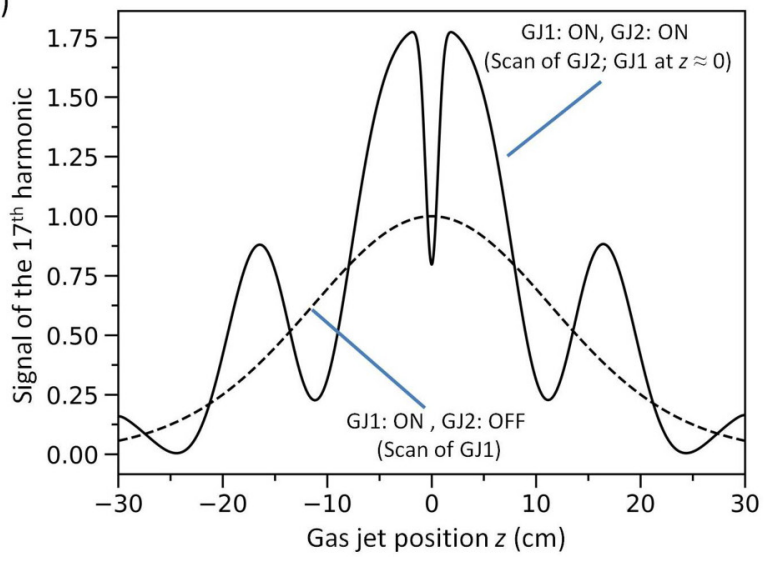

FIG. 4. Generation of 20-GW high-order harmonics using a dual gas jet. (a), (b) The upper panels shows the energy dependence of the XUV generated in Xe and Ar gas on the distance between GJ2 and GJ1 (placed at $z=0$ ). The error bars represent one standard deviation. The lower panels show the harmonic spectrum measured in the target area by recording the PE produced by the single photon of Ar gas. (c) Calculated yield of the 17th harmonic generated in Ar as a function of the distance between the gas jets (black solid line). The yield was calculated for $I_{\mathrm{L}} \approx 1.5 \times 10^{14} \mathrm{~W} \mathrm{~cm}^{-2}$ and $L \approx 1.5 \mathrm{~mm}$, $P \approx 25$ mbars for both jets. For comparison, the dependence of the XUV energy generated in a single Ar gas jet on the position of the GJ1 relative to the laser focus is shown (black dashed line).

introducing $\approx 98 \%$ losses. With the above given parameters, XUV intensities $I_{\text {XUV }}$ up to $\approx 7 \times 10^{15} \mathrm{~W} \mathrm{~cm}^{-2}$ have been reached and multiply charged ions $\left(\mathrm{Ar}^{n+}\right)$ with $n=1,2,3$ and 4 have been observed [Fig. 5(a)]. The signal of the single-charged 
ions $\left(\mathrm{Ar}^{+}, \mathrm{O}_{2}{ }^{+}, \mathrm{N}_{2}{ }^{+}\right.$, etc.) is proportional to the XUV pulse energy as it is generated by a single-photon ionization process. These ions are mainly produced away from the focus in the target area. The singly charged ion signal produced at the focus of the beam is considered negligible compared to the signal of the singly charged ions produced outside of the focus due to volume and single-photon ionization saturation effects which according to the lowest-order-perturbation theory are taking place for $I_{\mathrm{XUV}}>I_{\mathrm{XUV}}^{\text {(sat) }} \approx 7 \times 10^{12} \mathrm{~W} \mathrm{~cm}^{-2}$ [24]. The dependence of the $\mathrm{Ar}^{n+}$ (for $n \geqslant 2$ ) yield on $I_{\mathrm{XUV}}$ is shown in Fig. 5(b). In order to gain insight into the measured XUV intensity dependence of the $\mathrm{Ar}^{2+}$ and $\mathrm{Ar}^{3+}$ ion yields, we set up rate equations (described in the next section) accounting for all energetically allowed processes for ions up to $\mathrm{Ar}^{4+}$ [solid lines in Fig. 5(b)]. For the two-photon ionization of $\mathrm{Ar}$ we take the cross section equal to $10^{-51} \mathrm{~cm}^{4} \mathrm{~s}^{-1}$ (indicative two-photon ionization of a neutral atom [25]), while for all other two-photon-ionization processes we use a cross section of $10^{-52} \mathrm{~cm}^{4} \mathrm{~s}^{-1}$. However, we find that our results are robust for two-photon cross sections in the range from $10^{-51}$ to $10^{-53} \mathrm{~cm}^{4} \mathrm{~s}^{-1}$. For the three-photon processes we use a cross section of $10^{-85} \mathrm{~cm}^{6} \mathrm{~s}^{-2}$. The cross sections used in the numerical calculations are justified by the values deduced from the relevant saturation intensities $\left(I_{\mathrm{SAT}}\right)$ of the present paper (typical cross sections can be found in Refs. $[35,36]$ ). The measured $\mathrm{Ar}^{2+}$ signal after an increase with slope $s \approx 1.8 \pm$ 0.3 saturates at $I_{\mathrm{SAT}} \approx 2.2 \times 10^{15} \mathrm{~W} \mathrm{~cm}^{-2}$ where $s$ drops to $\approx 1.3 \pm 0.1$. The $\mathrm{Ar}^{3+}$ signal has a slope $s \approx 2.9 \pm 0.5$ and saturates at $I_{\mathrm{SAT}} \approx 3.2 \times 10^{15} \mathrm{~W} \mathrm{~cm}^{-2}$ with $s$ dropping to $\approx 1.7 \pm 0.2$. From the measured $I_{\mathrm{SAT}}$ we deduce $\mathrm{Ar}^{+}$twophoton and $\mathrm{Ar}^{2+}$ three-photon ionization cross sections to be $2 \times 10^{-52} \mathrm{~cm}^{4} \mathrm{~s}^{-1}$ and $1 \times 10^{-85} \mathrm{~cm}^{6} \mathrm{~s}^{-2}$, respectively, in good agreement with the values used in the calculations. Given the uncertainty factor of 2 in the intensity, the uncertainty factors of the measured cross sections are 4 and 9 , respectively. The $\mathrm{Ar}^{4+}$ signal appeared at intensities above $I_{\mathrm{SAT}}$ of $\mathrm{Ar}^{3+}$ and was at the detection limit not allowing any intensity dependence measurement. The corresponding values from the calculations are $s=1.8$ and $I_{\mathrm{SAT}} \approx 2.4 \times 10^{15} \mathrm{~W} \mathrm{~cm}^{-2}$ for $\mathrm{Ar}^{2+}$ and $s=3.1$ and $I_{\mathrm{SAT}} \approx 3.7 \times 10^{15} \mathrm{~W} \mathrm{~cm}^{-2}$ for $\mathrm{Ar}^{3+}$. Considering an uncertainty factor of $\approx 2$ (mainly associated with the measurement of the XUV focal spot size) in the estimation of $I_{\mathrm{XUV}}$, the results are in fair agreement. Figure 5(c) shows an excitation scheme that includes only the dominant processes. It is worth noting that comparing the results of our paper and those obtained using FEL sources [21] in the same spectral range one finds a striking difference in the intensity range of the two experiments. While in Ref. [21] $\mathrm{Ar}^{6+}$ is observed at intensities $1-2 \times 10^{14} \mathrm{~W} \mathrm{~cm}^{-2}$, in our paper charged states higher than $\mathrm{Ar}^{4+}$ are not observable at intensities $\sim 7 \times 10^{15} \mathrm{~W} \mathrm{~cm}^{-2}$. This difference can be attributed to interesting source dependent physics. The ionization yield for sequential processes depends linearly on the interaction volume and nonlinearly on the pulse duration depending on the number of sequential steps involved. The interaction volume in Ref. [21] (within the reported uncertainty) is the same or up to one order of magnitude larger than in this paper and the pulse duration is $100 \mathrm{fs}$, i.e., one order of magnitude larger. For the generation of $\mathrm{Ar}^{4+}$ a sequential process of three steps is necessary (and for the generation of $\mathrm{Ar}^{6+}$ another two). (a)

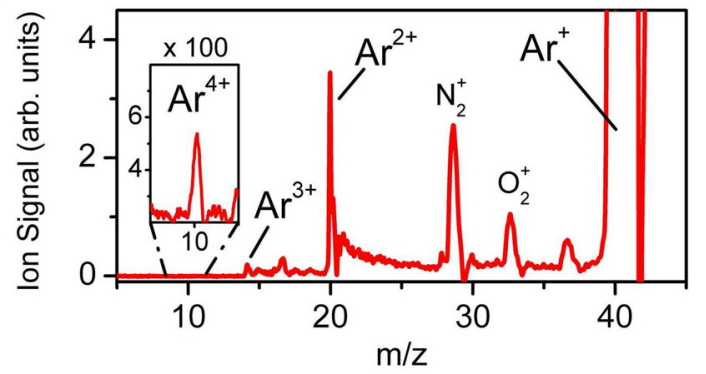

(b)
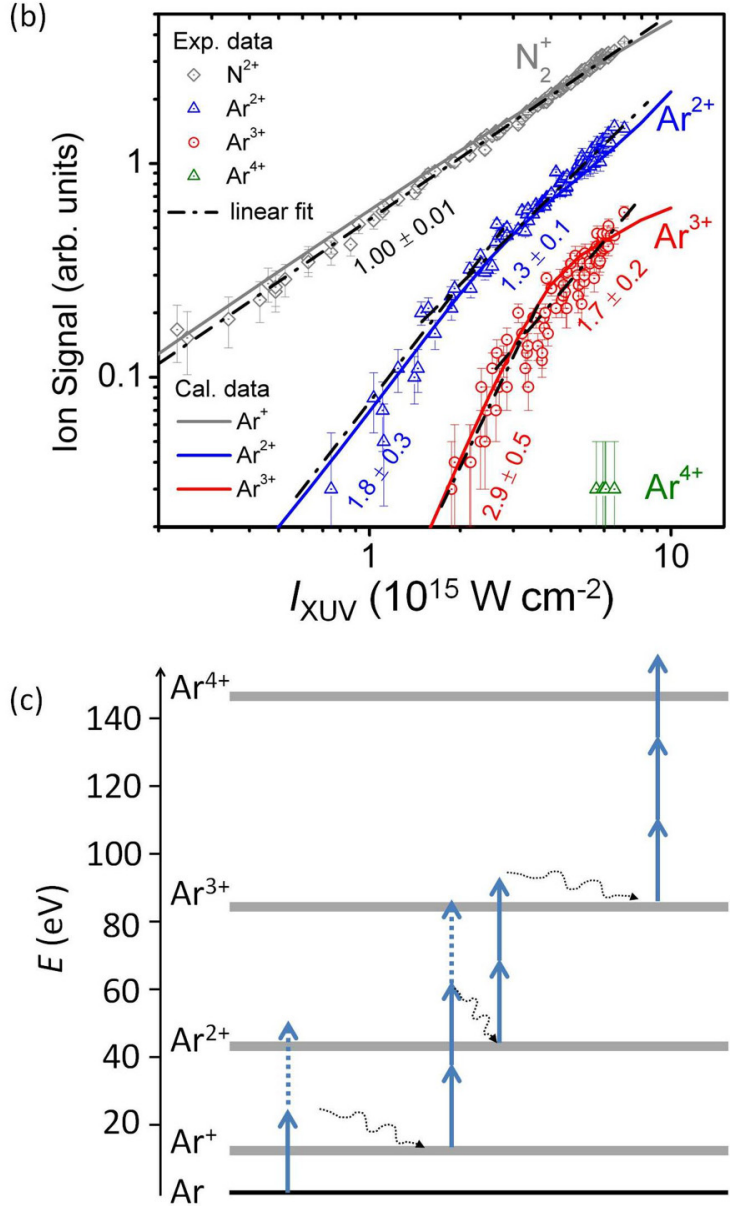

FIG. 5. Multiple ionization of argon atoms using the 20-GW high-order harmonic source. (a) TOF mass spectrum produced by the interaction of the focused 11th-15th harmonics with argon. The spectrum shows multiple charged $\mathrm{Ar}$ ions $\left(\mathrm{Ar}^{n+}\right)$ with $n$ up to 4 . (b) Dependence of the $\mathrm{Ar}^{2,3+}$ yield on the $I_{\mathrm{XUV}}$. For calibrating the XUV energy ( $x$ axis) the $\mathrm{O}_{2}{ }^{+}$signal was used. The black dashed lines show a linear fit on the raw data. The error bars represent one standard deviation of the mean. The solid lines show the results of the numerical calculations, including volume integration. As is expected from a single-photon ionization process, the dependence of the calculated $\mathrm{Ar}^{+}$yield on $I_{\mathrm{XUV}}$ is linear and matches well with the experimental data of $\mathrm{N}_{2}^{+}$. As the calculated $\mathrm{Ar}^{+}$yield (gray solid line) is orders of magnitude higher than the $\mathrm{Ar}^{2+}$ yield, for visualization reasons, the calculated $\mathrm{Ar}^{+}$signal was divided by a factor of $8 \times 10^{3}$, and the measured $\mathrm{Ar}^{+}$signal was normalized to it. In arbitrary units measured, $\mathrm{Ar}^{2+}, \mathrm{Ar}^{3+}$, and $\mathrm{Ar}^{4+}$ yield points are normalized to one and the same calculated single ion yield point. (c) Multi-XUV-photon multiple ionization scheme [excluding higher-order processes (ATI)] of Ar which supports the obtained results. 
These parameters of Ref. [21] would produce three to four orders-of-magnitude higher $\mathrm{Ar}^{4+}$ yield for the same intensity as in our experiment. This is not enough to explain the intensity difference in the two experiments for the processes involved. However, it is well established that the yield of multiphoton processes depends on the coherence properties of the radiation used. Thus, for an $N$ photon process, the yield is proportional to $N$ ! for thermal (chaotic) radiation, proportional to $(2 N-1)$ !! for squeezed vacuum states of light or proportional to unity for coherent radiation. The multiphoton yield dependence on the coherence properties of the light was theoretically shown already in the 1970s [37-39] and experimentally verified in 1974 [40] and more recently in Ref. [41]. It is also well established that FEL radiation is incoherent, unless seeded by laser harmonics, while harmonic radiation is coherent. Thus, for the many photon processes involved in this paper and in Ref. [21] even partial validity of the $N$ ! dependence (due to not completely incoherent FEL radiation and not $100 \%$ coherent harmonic radiation) results in a much higher yield in Ref. [21] due to the incoherence of the source used. Hence the comparison of Ref. [21] with the present paper is compatible with the coherence properties of the different sources used. It also highlights the importance of the present achievement exactly because harmonic sources do not favor multiphoton processes in terms of yield.

\section{NUMERICAL CALCULATIONS}

The numerical calculations provide significant information about the individual contributing ionization channels by computing the ion yields of each allowed pathway contributing to the formation of $\mathrm{Ar}^{2+}$ and $\mathrm{Ar}^{3+}$. In the calculations we use a Gaussian laser pulse of FWHM equal to $\tau_{\mathrm{XUV}}=10 \mathrm{fs}$. Moreover, we perform a volume average for our results for the ion yields. We do so as follows. First, for a certain peak intensity we used the following equation [36] to determine the intensity, $I_{\mathrm{XUV}}$, at each point $(r, z)$ in cylindrical coordinates:

$$
I_{\mathrm{XUV}}(r, z ; t)=I_{\mathrm{XUV}}(t) \frac{w_{0}^{2}}{w(z)^{2}} \exp \left[-\frac{2 r^{2}}{w(z)^{2}}\right]
$$

where $r$ is the radius and $z$ is the beam propagation axis. $w(z)$ is the beam radius, defined in terms of the beam waist, $w_{0}=$ $1 \mu \mathrm{m}$, and the Rayleigh length, $z_{\mathrm{R}}=51.5 \mu \mathrm{m}$, as

$$
w(z)=w_{0} \sqrt{1+\left(z / z_{R}\right)^{2}} .
$$

We calculated the ion yield in a volume with limits $r_{\max }=$ $3 \mathrm{~mm}$ in the radial direction and $z_{\min }=-3 \mathrm{~mm}$ to $z_{\max }=$ $3 \mathrm{~mm}$ in the $z$ direction. These ion yields were then integrated using the following expression [42]:

$$
P_{i}=\int_{0}^{r_{\max }} \int_{z_{\min }}^{z_{\max }} 2 \pi r N_{i}(r, z) d z d r
$$

where $P_{i}$ is the yield of the ion $i$ integrated over the volume and $N_{i}$ is the yield of ion $i$. We have checked that our results for $P_{i}$ converge. The yields for the main pathways leading to the formation of $\mathrm{Ar}^{2+}$ and $\mathrm{Ar}^{3+}$ are not volume integrated. In our computations the three-photon transition is energetically allowed when the photon energy is equal to or above $22 \mathrm{eV}$.
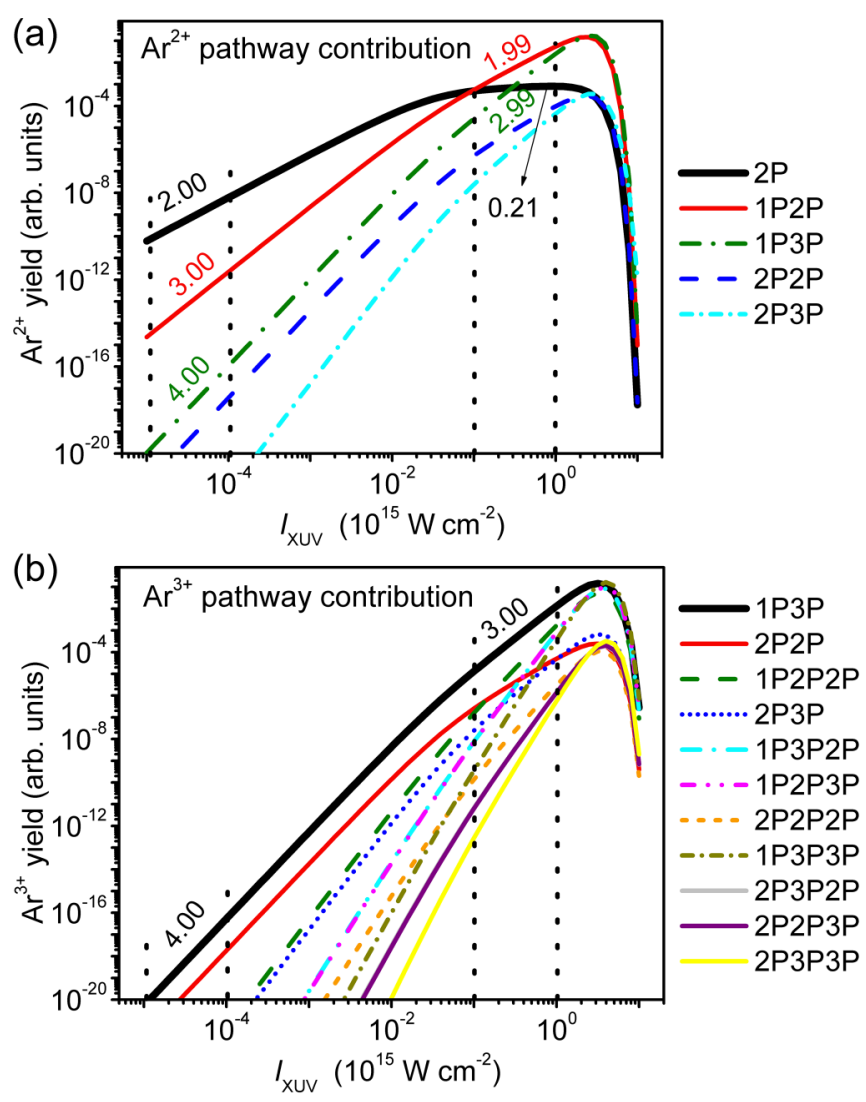

FIG. 6. Calculated ionization yields of different ionization pathways. These calculations are performed for a central photon energy of $22 \mathrm{eV}$ and a pulse duration of $10 \mathrm{fs}$, and no volume integration has been included. (a) shows the yield of $\mathrm{Ar}^{2+}$ as a function of the XUV intensity. The black thick line is the yield of the two-photon DDI of the Ar pathway. All other curves denoted $n \operatorname{PmP}(m=1,2 ; n=2,3)$ refer to the sequential pathways of $n$-photon ionization of Ar followed by $m$-photon ionization of $\mathrm{Ar}^{+}$. From this graph we deduce that below saturation the two-photon DDI is the dominant pathway, while after saturation the lowest-order sequential process prevails. (b) shows the XUV intensity dependence of the $\mathrm{Ar}^{3+}$ yield. The black thick line is the single-photon ionization of Ar followed by three-photon direct ejection of two electrons from $\mathrm{Ar}^{+}$, which is the dominant pathway at all intensities, while all other curves denoted $h P i P j P$ refer to the sequential processes of $h$-photon $(h=1,2)$ ionization of Ar followed by $i$-photon ionization $(i=2,3)$ of $\mathrm{Ar}^{+}$and eventually by $j$-photon ionization $(j=2,3)$ of $\mathrm{Ar}^{2+}$.

We find that our results do not change when the photon energy changes from 22 to $23.3 \mathrm{eV}$, which is the maximum energy considered in the experiment. However, if we reduce the photon energy below $22 \mathrm{eV}$ then the sequential process of one-photon ionization of $\mathrm{Ar}$ followed by three-photon ionization of $\mathrm{Ar}^{+}$(1P3P) is not energetically allowed and the slope we obtain for the $\mathrm{Ar}^{3+}$ ion yield is closer to 4 . We note that in our computations all energies are obtained with MOLPRO (a quantum chemistry package) using Hartree-Fock with a 6-311-G basis. We have used the same basis for previous FEL processes in $\mathrm{Ar}$ [43]. Figure 6(a) shows these yields for $\mathrm{Ar}^{2+}$ formation as a function of the XUV intensity. The black line is the yield of the two-photon direct-double-ionization (DDI) 
channel. All other curves denoted $n P m P(m=1,2$ and $n=$ $2,3)$ refer to the sequential pathways of $n$-photon ionization of Ar followed by $m$-photon ionization of $\mathrm{Ar}^{+}$. Before saturation of the $\mathrm{Ar}^{+}$signal the dominant process for the $\mathrm{Ar}^{2+}$ production is the two-photon DDI, while upon saturation the sequential processes set in and eventually prevail. In Fig. 6(b) several different channels producing $\mathrm{Ar}^{3+}$ are compared. The notation $h \operatorname{PiPjP}(h=1,2 ; i=2,3$; and $j=2,3)$ stands for $h$-photon ionization of Ar, followed by $i$-photon DDI of $\mathrm{Ar}^{+}$or $i$-photon ionization of $\mathrm{Ar}^{+}$followed by $j$-photon ionization of $\mathrm{Ar}^{2+}$. The dominating mechanism for the production of $\mathrm{Ar}^{3+}$ is the single-photon ionization of Ar followed by a three-photon DDI of $\mathrm{Ar}^{+}$. The slope of 4 of this four-photon process reduces to 3 in the intensity region of the experiment as in this region the Ar single-photon ionization is saturated.

\section{CONCLUSIONS}

This paper demonstrates the most powerful gas target HHG source ever, emitting XUV pulses with a pulse energy as high as published XUV pulse energies of FEL sources in the spectral region $17-30 \mathrm{eV}$. The unprecedented XUV intensities achieved with this source are evidenced by the observation of multi-XUV-photon multiple ionization of argon through a series of sequential and direct ionization processes as proven by numerical calculations. A comparison between HHG and FEL induced multiple ionization reveals differences that can be attributed to the different temporal and coherence characteristics of the two source types. The paper establishes that table-top HHG sources reached intensities allowing the study and exploitation of highly nonlinear XUV processes [44,45], advancing the capacities of ultrafast XUV science $[10,33,46]$. Besides the prospects opened in small and intermediate size laboratories the present results are highly relevant to the mission of the under-implementation Extreme Light Infrastructure - Attosecond Light Pulse Source
(ELI-ALPS) facility. Its GHHG SYLOS compact beam line [47] uses a similar arrangement operating at $1-\mathrm{kHz}$ repetition rate operation. The XUV pulse energy levels of the present paper at $1-\mathrm{kHz}$ repetition rate and isolated pulse operation open up unique prospects for few-body coincidence experiments in nonlinear XUV laser-matter interactions exploited in detailed studies of ultrafast dynamics.

\section{ACKNOWLEDGMENTS}

We acknowledge support of this work by the LASERLABEUROPE (EC's Seventh Framework Programme Grant No. 284464), H2020-INFRAIA project NFFA-Europe (Grant No. 654360), "HELLAS-CH" (MIS Grant No. 5002735) [which is implemented under the "Action for Strengthening Research and Innovation Infrastructures," funded by the Operational Program "Competitiveness, Entrepreneurship and Innovation" (NSRF 2014-2020) and cofinanced by Greece and the European Union (European Regional Development Fund)], and the European Union's Horizon 2020 research and innovation program under Marie Sklodowska-Curie MEDEA Grant No. 641789. ELI-ALPS is supported by the European Union and cofinanced by the European Regional Development Fund (GINOP Grant No. 2.3.6-15-2015-00001). We thank G. Konstantinidis and G. Deligiorgis from the Materials and Devices Division of FORTH-IESL for their support in maintaining the quality of the optical components, N. Papadakis for developing the electronic devices used in the beam line, and S. Karsch from Max Plank Institute for Quantum Optics for his assistance on maintaining the performance of the Ti:S laser compressor. Also, A.N. (on leave from ELI-ALPS, presently employed at FORTH) and P.T. (consultant member of ELIALPS) thank ELI-ALPS for support and fruitful collaboration.

A.N., I. O., I.M., M.D., and S.K. contributed equally to this paper.
[1] M. Goeppert-Mayer, Ann. Phys. (Leipzig). 9, 273 (1931).

[2] G. S. Voronov and N. B. Delone, JETP Lett. 1, 66 (1965).

[3] Y. Kobayashi, T. Sekikawa, Y. Nabekawa, and S. Watanabe, Opt. Lett. 23, 64 (1998).

[4] P. Tzallas, E. Skantzakis, L. A. A. Nikolopoulos, G. D. Tsakiris, and D. Charalambidis, Nat. Phys. 7, 781 (2011).

[5] M. Lewenstein, P. Balcou, M. Y. Ivanov, A. L'Huillier, and P. B. Corkum, Phys. Rev. A 49, 2117 (1994).

[6] C. I. Blaga, F. Catoire, P. Colosimo, G. G. Paulus, H. G. Muller, P. Agostini and L. F. DiMauro, Nat. Phys. 5, 335 (2009).

[7] N. Tsatrafyllis, I. K. Kominis, I. A. Gonoskov, and P. Tzallas, Nat. Commun. 8, 15170 (2017).

[8] S. Chatziathanasiou, S. Kahaly, E. Skantzakis, G. Sansone, R. Lopez-Martens, S. Haessler, K. Varju, G. Tsakiris, D. Charalambidis, and P. Tzallas, Photonics 4, 26 (2017).

[9] C. M. Heyl, C. L. Arnold, A. Couairon, and A. L'Huillier, J. Phys. B 50, 013001 (2017).

[10] M. Reduzzi, P. Carpeggiani, S. Kühn, F. Calegari, M. Nisoli, S. Stagira, C. Vozzi, P. Dombi, S. Kahaly, P. Tzallas, D. Charalambidis, K. Varju, K. Osvay, and G. Sansone, J. Electron Spectros. Relat. Phenomena 204, 257 (2015).

[11] F. Krausz and M. Ivanov, Rev. Mod. Phys. 81, 163 (2009).
[12] P. B. Corkum and F. Krausz, Nat. Phys. 3, 381 (2007).

[13] T. Gaumnitz, A. Jain, Y. Pertot, M. Huppert, I. Jordan, F. ArdanaLamas, and H. J. Wörner, Opt. Express 25, 27506 (2017).

[14] K. Zhao, Q. Zhang, M. Chini, Y. Wu, X. Wang, and Z. Chang, Opt. Lett. 37, 3891 (2012).

[15] A. A. Sorokin, S. V. Bobashev, T. Feigl, K. Tiedtke, H. Wabnitz, and M. Richter, Phys. Rev. Lett. 99, 213002 (2007).

[16] L. Young, E. P. Kanter, B. Krässig, Y. Li, A. M. March, S. T. Pratt, R. Santra, S. H. Southworth, N. Rohringer, L. F. DiMauro, G. Doumy, C. A. Roedig, N. Berrah, L. Fang, M. Hoener, P. H. Bucksbaum, J. P. Cryan, S. Ghimire, J. M. Glownia, D. A. Reis, J. D. Bozek, C. Bostedt, and M. Messerschmidt, Nature (London) 466, 56 (2010).

[17] B. Rudek, S.-K. Son, L. Foucar, S. W. Epp, B. Erk, R. Hartmann, M. Adolph, R. Andritschke, A. Aquila, N. Berrah, C. Bostedt, J. Bozek, N. Coppola, F. Filsinger, H. Gorke, T. Gorkhover, H. Graafsma, L. Gumprecht, A. Hartmann, G. Hauser, S. Herrmann, H. Hirsemann, P. Holl, A. Hömke, L. Journel, C. Kaiser, N. Kimmel, F. Krasniqi, K.-U. Kühnel, M. Matysek, M. Messerschmidt, D. Miesner, T. Möller, R. Moshammer, K. Nagaya, B. Nilsson, G. Potdevin, D. Pietschner, C. Reich, D. Rupp, G. Schaller, I. Schlichting, C. Schmidt, F. Schopper, 
S. Schorb, C.-D. Schröter, J. Schulz, M. Simon, H. Soltau, L. Strüder, K. Ueda, G. Weidenspointner, R. Santra, J. Ullrich, A. Rudenko, and D. Rolles, Nat. Photon. 6, 858 (2012).

[18] H. Fukuzawa, S.-K. Son, K. Motomura, S. Mondal, K. Nagaya, S. Wada, X.-J. Liu, R. Feifel, T. Tachibana, Y. Ito, M. Kimura, T. Sakai, K. Matsunami, H. Hayashita, J. Kajikawa, P. Johnsson, M. Siano, E. Kukk, B. Rudek, B. Erk, L. Foucar, E. Robert, C. Miron, K. Tono, Y. Inubushi, T. Hatsui, M. Yabashi, M. Yao, R. Santra, and K. Ueda, Phys. Rev. Lett. 110, 173005 (2013).

[19] W. Ackermann, G. Asova, V. Ayvazyan, A. Azima, N. Baboi, J. Bähr, V. Balandin, B. Beutner, A. Brandt, A. Bolzmann, R. Brinkmann, O. I. Brovko, M. Castellano, P. Castro, L. Catani, E. Chiadroni, S. Choroba, A. Cianchi, J. T. Costello, D. Cubaynes, J. Dardis, W. Decking, H. Delsim-Hashemi, A. Delserieys, G. Di Pirro, M. Dohlus, S. Düsterer, A. Eckhardt, H. T. Edwards, B. Faatz, J. Feldhaus, K. Flöttmann, J. Frisch, L. Fröhlich, T. Garvey, U. Gensch, C. Gerth, M. Görler, N. Golubeva, H.-J. Grabosch, M. Grecki, O. Grimm, K. Hacker, U. Hahn, J. H. Han, K. Honkavaara, T. Hott, M. Hüning, Y. Ivanisenko, E. Jaeschke, W. Jalmuzna, T. Jezynski, R. Kammering, V. Katalev, K. Kavanagh, E. T. Kennedy, S. Khodyachykh, K. Klose, V. Kocharyan, M. Körfer, M. Kollewe, W. Koprek, S. Korepanov, D. Kostin, M. Krassilnikov, G. Kube, M. Kuhlmann, C. L. S. Lewis, L. Lilje, T. Limberg, D. Lipka, F. Löhl, H. Luna, M. Luong, M. Martins, M. Meyer, P. Michelato, V. Miltchev, W. D. Möller, L. Monaco, W. F. O. Müller, O. Napieralski, O. Napoly, P. Nicolosi, D. Nölle, T. Nuñez, A. Oppelt, C. Pagani, R. Paparella, N. Pchalek, J. Pedregosa-Gutierrez, B. Petersen, B. Petrosyan, G. Petrosyan, L. Petrosyan, J. Pflüger, E. Plönjes, L. Poletto, K. Pozniak, E. Prat, D. Proch, P. Pucyk, P. Radcliffe, H. Redlin, K. Rehlich, M. Richter, M. Roehrs, J. Roensch, R. Romaniuk, M. Ross, J. Rossbach, V. Rybnikov, M. Sachwitz, E. L. Saldin, W. Sandner, H. Schlarb, B. Schmidt, M. Schmitz, P. Schmüser, J. R. Schneider, E. A. Schneidmiller, S. Schnepp, S. Schreiber, M. Seidel, D. Sertore, A. V. Shabunov, C. Simon, S. Simrock, E. Sombrowski, A. A. Sorokin, P. Spanknebel, R. Spesyvtsev, L. Staykov, B. Steffen, F. Stephan, F. Stulle, H. Thom, K. Tiedtke, M. Tischer, S. Toleikis, R. Treusch, D. Trines, I. Tsakov, E. Vogel, T. Weiland, H. Weise, M. Wellhöfer, M. Wendt, I. Will, A. Winter, K. Wittenburg, W. Wurth, P. Yeates, M. V. Yurkov, I. Zagorodnov, and K. Zapfe, Nat. Photon. 1, 336 (2007).

[20] S. Serkez, G. Geloni, S. Tomin, G. Feng, E. V. Gryzlova, A. N. Grum-Grzhimailo, and M. Meyer, J. Opt. 20, 24005 (2018).

[21] K. Motomura, H. Fukuzawa, L. Foucar, X.-J. Liu, G. Prümper, K. Ueda, N. Saito, H. Iwayama, K. Nagaya, H. Murakami, M. Yao, A. Belkacem, M. Nagasono, A. Higashiya, M. Yabashi, T. Ishikawa, H. Ohashi, and H. Kimura, J. Phys. B 42, 221003 (2009).

[22] C. Altucci, C. Beneduce, R. Bruzzese, C. de Lisio, G. S. Sorrentino, T. Starczewski, and F. Vigilante, J. Phys. D: Appl. Phys. 29, 68 (1996).

[23] E. J. Takahashi, H. Hasegawa, Y. Nabekawa, and K. Midorikawa, Opt. Lett. 29, 507 (2004).

[24] N. Tsatrafyllis, B. Bergues, H. Schroder, L. Veisz, E. Skantzakis, D. Gray, B. Bodi, S. Kuhn, G. D. Tsakiris, D. Charalambidis, and P. Tzallas, Sci. Rep. 6, 21556 (2016).

[25] J. A. R. Samson and W. C. Stolte, J. Electron Spectros. Relat. Phenomena 123, 265 (2002).
[26] Y. Tao, S. J. Goh, H. M. J. Bastiaens, P. J. M. van der Slot, S. G. Biedron, S. V. Milton, and K.-J. Boller, Opt. Express 25, 3621 (2017).

[27] E. Constant, D. Garzella, P. Breger, E. Mével, C. Dorrer, C. Le Blanc, F. Salin, and P. Agostini, Phys. Rev. Lett. 82, 1668 (1999).

[28] K. Midorikawa, Y. Nabekawa, and A. Suda, Prog. Quantum Electron. 32, 43 (2008).

[29] J. Seres, V. S. Yakovlev, E. Seres, C. Streli, P. Wobrauschek, C. Spielmann, and F. Krausz, Nat. Phys. 3, 878 (2007).

[30] D. E. Laban, A. J. Palmer, W. C. Wallace, N. S. Gaffney, R. P. M. J. W. Notermans, T. T. J. Clevis, M. G. Pullen, D. Jiang, H. M. Quiney, I. V. Litvinyuk, D. Kielpinski, and R. T. Sang, Phys. Rev. Lett. 109, 263902 (2012).

[31] E. J. Takahashi, Y. Nabekawa, and K. Midorikawa, Opt. Lett. 27, 1920 (2002).

[32] E. J. Takahashi, Y. Nabekawa, T. Otsuka, M. Obara, and K. Midorikawa, Phys. Rev. A 66, 021802(R) (2002).

[33] P. Tzallas, B. Bergues, D. Rompotis, N. Tsatrafyllis, S. Chatziathanassiou, A. Muschet, L. Veisz, H. Schröder, and D. Charalambidis, J. Opt. 20, 24018 (2018).

[34] C.-G. Wahlstrom, J. Larsson, A. Persson, T. Starczewski, S. Svanberg, P. Saliéres, P. Balcou, and A. L'Huillier, Phys. Rev. A 48, 4709 (1993).

[35] P. Lambropoulos, Comments At. Mol. Phys. 20, 199 (1987).

[36] G. M. Nikolopoulos and P. Lambropoulos, J. Phys. B 47, 115001 (2014).

[37] P. Lambropoulos, Advances in Atomic and Molecular Physics (Academic, New York, 1976), Vol. 12, p. 87.

[38] A. T. Georges and P. Lambropoulos, Phys. Rev. A 20, 991 (1979).

[39] G. M. Nikolopoulos and P. Lambropoulos, Phys. Rev. A 86, 033420 (2012).

[40] C. Lecompte, G. Mainfray, C. Manus, and F. Sanchez, Phys. Rev. Lett. 32, 265 (1974).

[41] Kirill Yu. Spasibko, Denis A. Kopylov, Victor L. Krutyanskiy, Tatiana V. Murzina, Gerd Leuchs, and Maria V. Chekhova, Phys. Rev. Lett. 119, 223603 (2017).

[42] E. T. Karamatskos, D. Markellos, and P. Lambropoulos, J. Phys. B 46, 164011 (2014).

[43] A. O. G. Wallis, L. Lodi, and A. Emmanouilidou, Phys. Rev. A 89, 063417 (2014).

[44] P. Lambropoulos, K. G. Papamihail, and P. Decleva, J. Phys. B 44, 175402 (2011).

[45] M. G. Makris, P. Lambropoulos, and A. Mihelič, Phys. Rev. Lett. 102, 033002 (2009).

[46] E. Foumouo, S. Laulan, B. Piraux, and H. Bachau, J. Phys. B 39, S427 (2006).

[47] S. Kühn, M. Dumergue, S. Kahaly, S. Mondal, M. Füle, T. Csizmadia, B. Farkas, B. Major, Z. Várallyay, E. Cormier, M. Kalashnikov, F. Calegari, M. Devetta, F. Frassetto, E. Månsson, L. Poletto, S. Stagira, C. Vozzi, M. Nisoli, P. Rudawski, S. Maclot, F. Campi, H. Wikmark, C. L. Arnold, C. M. Heyl, P. Johnsson, A. L'Huillier, R. Lopez-Martens, S. Haessler, M. Bocoum, F. Boehle, A. Vernier, G. Iaquaniello, E. Skantzakis, N. Papadakis, C. Kalpouzos, P. Tzallas, F. Lépine, D. Charalambidis, K. Varjú, K. Osvay, and G. Sansone, J. Phys. B 50, 132002 (2017). 